\title{
The Dynamo Software Package for Cryo-electron Tomography and Subtomogram Averaging
}

\author{
Paula Navarro ${ }^{1}$, Stefano Scaramuzza $^{2}$, Henning Stahlberg $^{2}$ and Daniel Castaño-Díez ${ }^{2}$ \\ ${ }^{1}$ MGH - Harvard Medical School, Boston, Massachusetts, United States, ${ }^{2}$ University of Basel, Basel, \\ Basel-Stadt, Switzerland
}

Cryo-electron tomography (cryo-ET) and subtomogram averaging (STA) have become popular techniques for structural determination of biological macrocomplexes in their native environment. CryoET allows three-dimensional (3D) visualization of cellular organelles and macromolecular complexes at nanometer resolution ${ }^{1}$ in their close-to-native environment. Briefly, in cryo-ET, the specimen is physically rotated around a single axis inside the electron microscope. This axis is perpendicular to the optical axis of the microscope, and thus, to the direction of the electron beam. Micrographs are collected at each rotation angle, resulting in a set of tilted micrographs referred to as tilt series. Each micrograph represents a view of the specimen at different tilt angles. The tilt series is then computationally aligned and reconstructed into a 3D tomogram. Unfortunately, the rotation possible, i.e., the tilt range, is physically restricted by the microscope geometry, thus, tilt series are usually acquired from $-60^{\circ}$ to $+60^{\circ}$. As a result, reconstructed tomograms experience partial sampling or missing information in Fourier Space. The missing information exhibits a characteristic wedge-like shape, which is known as the missing wedge. In real space, the missing wedge manifests itself as a blurred elongation of the imaged specimen in the direction of the electron beam ${ }^{2}$.

STA was conceived as an image processing method that computationally manages image processing for cryo-ET datasets to obtain signal-enhanced 3D structures ${ }^{3,4}$. Commonly, biological macrocomplexes, present as multiple copies within a tomogram, are computationally extracted into subvolumes, also defined as subtomograms. Subsequently, STA perform 3D alignment to compute a final average of such subtomograms retrieving a signal-enhanced 3D density map of the target macromolecular complex. Importantly, the resulting 3D density map provides information concerning the spatial distribution, orientation and interactions of the target macromolecular complex with their biological microenvironment. Additionally, STA aims to overcome by computational means typical limitations linked to cryo-ET, such as the missing wedge, sample geometry and low signal-to-noise ration among others $^{5}$. Generally, data processing pipelines for cryo-ET and STA are well-established, currently aiming to improve automation, data management and performance of CPU and GPU algorithms to accommodate large data sets.

Dynamo is a software package specialized in image processing for cryo-ET and STA that acts as a modular toolbox adaptable to different biological scenarios, allowing custom designed pipelines for STA ${ }^{6-9}$. Dynamo is broadly used in a plethora of cryo-ET and STA projects ranging from high-resolution STA ${ }^{10-}$ 12 to analysis of cellular sociology in cryo-focused ion beam datasets ${ }^{13}$. Since STA highly depends on the quality of the tilt series alignment, we have implemented tools to integrate tomographic alignment of tilt series and STA in Dynamo.

With the advent of modern technologies for cryo-ET, high-quality tilt series are more rapidly acquired than processed and analyzed. Importantly, tilt series alignment is an essential step in cryo-ET and STA, 
which has a direct impact on the quality of the 3D reconstructed tomogram, and therefore, limits attainable resolution in STA. While different software packages have made available several approaches for automated marker-based alignment of tilt series, manual user intervention remains indispensable for many datasets, thus, preventing high-throughput tomography. Consequently, we have implemented a tilt series alignment framework for cryo-ET where fiducial markers are automatically detected and tracked to generate a projection model with minimal input parameters needed from the users. This approach combined with Dynamo repository - our new batching system for tilt series datasets -, allows highthroughput unsupervised alignment of tilt series. The new module extends Dynamo with a large repertory of tools for tomographic alignment and reconstruction, as specific visualization browsers and localized reconstruction systems. Finally, our image processing pipeline enables users to rapidly assess the biological relevance of specific dataset preceding high-throughput in situ cryo-ET and STA.

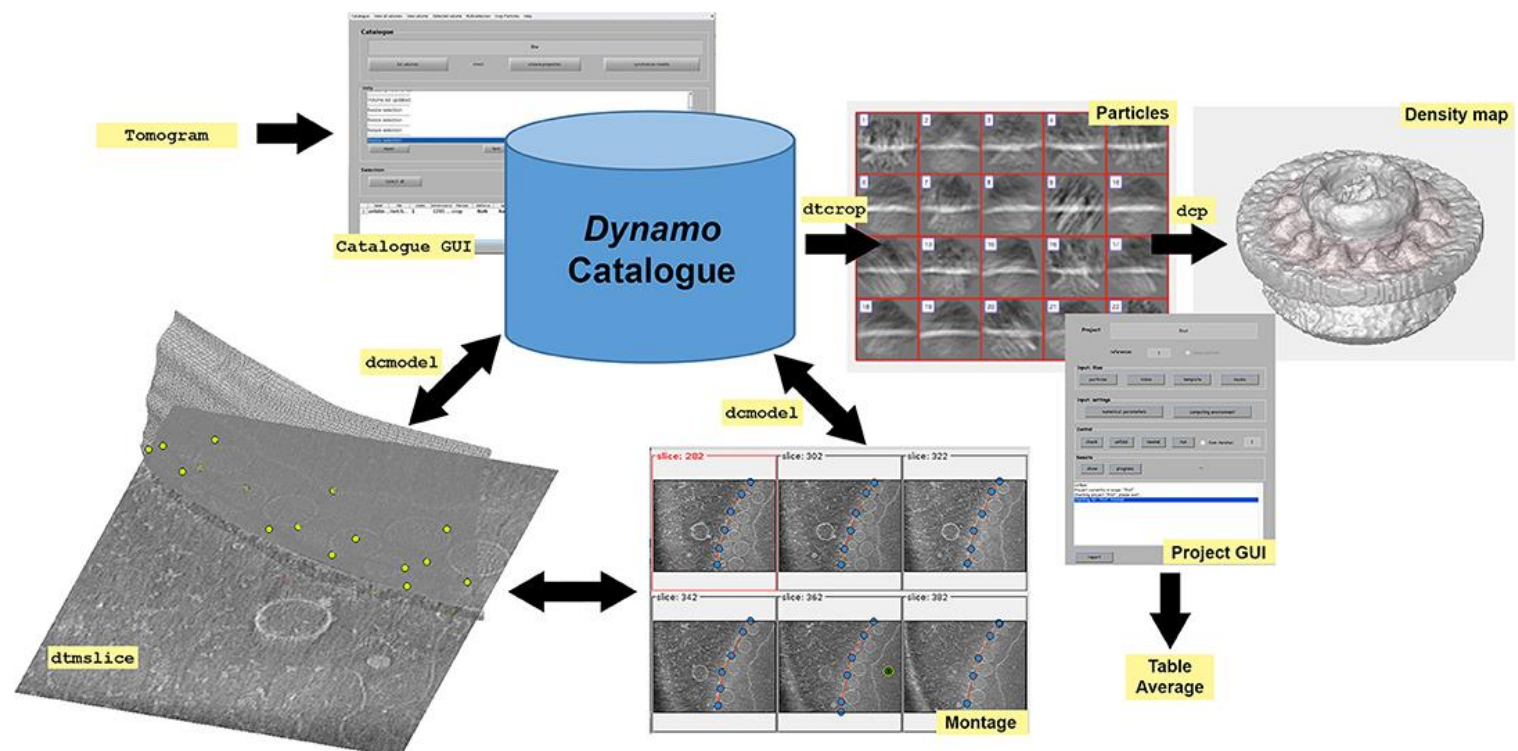

Figure 1. Graphical Abstract. Dynamo workflow. Dynamo catalogue and its GUI (dynamo_catalogue_manager or $\mathrm{dcm}$ ) provide a robust and simple way to keep track of the transformations and annotations performed on tomographic data sets. The aim is to allow the definition of positions (particles) inside each tomogram, so that subvolumes can be defined and cropped, to produce a unique data folder with its corresponding table, which can then be used to feed a project for alignment or classification. The catalogue stores both raw data and annotations performed on the data. In Dynamo, annotated tomographic data is defined as geometrical models, therefore Dynamo refers to them with the name model. Dynamo catalogue keeps track of volumetric processing tasks performed on the tomogram (such as binning or flipping) as well as all manipulations for particle modelling, orientation and geometry. Furthermore, tomogram properties registered in the catalogue include paths with the file location, defocus, magnification and a description of the missing wedge among other things. Tomographic data is annotated in dtmslice GUI with the help of montage tools that allow the optimal handling and representation of userpositioned points defined in Dynamo models, dcmodel. Particle positioning can be defined by clicking directly onto the particles or using semi-automation tools integrated in GUIs as dtmslice and montage. Particles are extracted from the models using dtcrop to obtain a data folder with the extracted particles and a table that contains the metadata information of the extracted particles. Particles are usually averaged to generate a first template. Then, particles, table and template are used to feed an STA project in the dcp GUI. After running an STA project, an average of the particles and a corresponding table are generated. The density map of the average can be visualized with different volume rendering tools. 


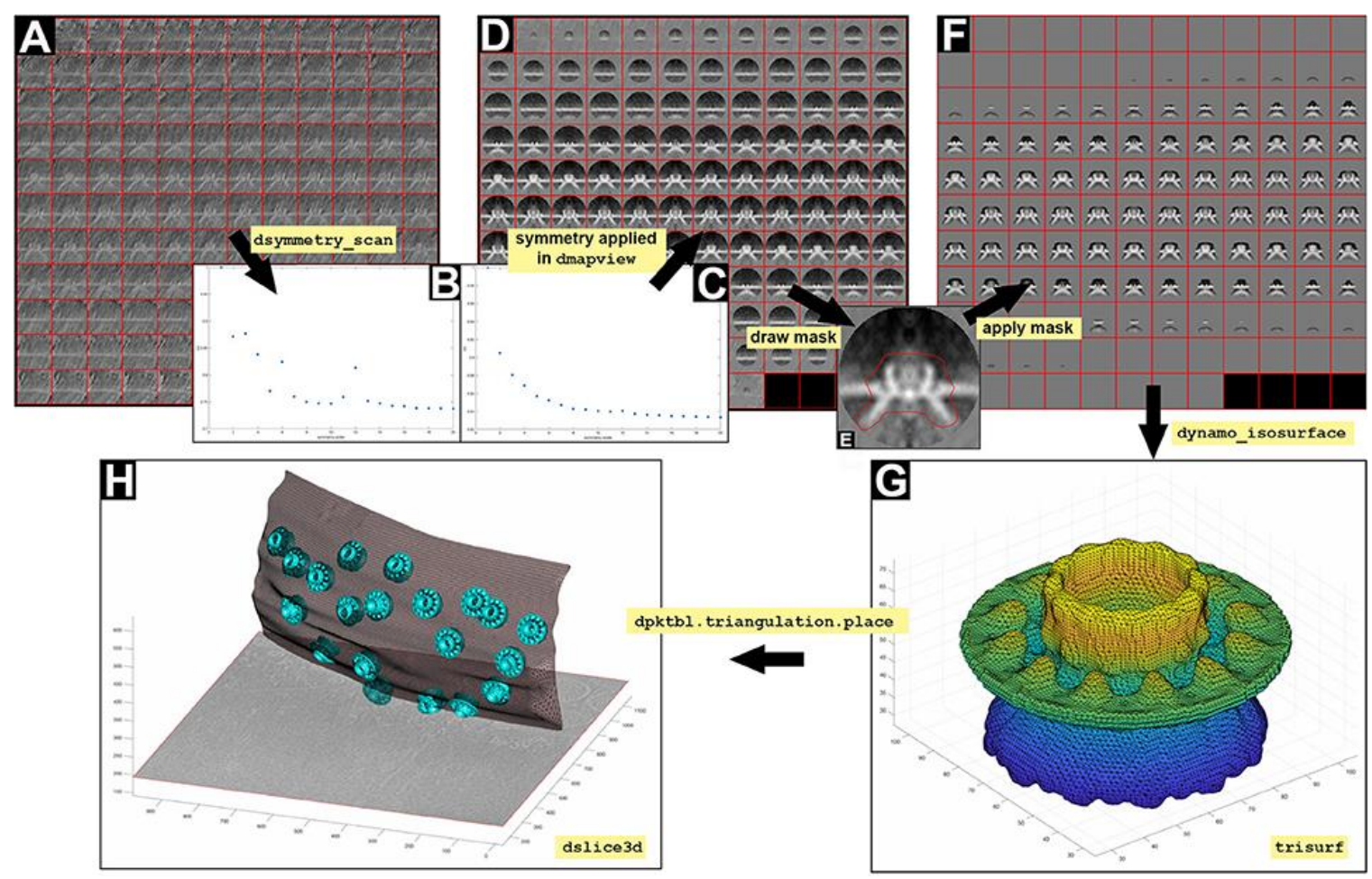

Figure 2. Visualization of 3D scenes from Dynamo models and density maps. (A) Y view of the average obtained by executing the STA project localized. (B-C) Graphical representation of symmetry (symmetry_scan) found in A with and without applying the mask file teeth, respectively. (D) Y view of the $\mathrm{c} 12$ symmetrized average obtained by executing the STA project. (E) Y view of the slice number 65 of the average obtained from STA. The mask is defined by manually drawing an area covering the crown structure (red line). (F) Y view of the c12 symmetrized STA average after application of the mask defined in E. (G) Graphical reconstruction using the function trisurf on the 'crown' structure displayed as a triangulated surface (dynamo_isosurface function). (H) 3D crown average displayed (dslice3d function) in cyan inserted in the OMM (grey triangulated mesh by dpktbl.triangulation.place) present in this tomogram.

References

1. Leigh, K. E. et al. Subtomogram averaging from cryo-electron tomograms. Methods Cell Biol. 152, 217-259 (2019).

2. Lučić, V., Rigort, A. \& Baumeister, W. Cryo-electron tomography: The challenge of doing structural biology in situ. J. Cell Biol. 202, 407 (2013).

3. Briggs, J. A. G. Structural biology in situ--the potential of subtomogram averaging. Curr. Opin. Struct. Biol. 23, 261-267 (2013).

4. Wan, W. \& Briggs, J. a. G. Cryo-Electron Tomography and Subtomogram Averaging. Methods Enzymol. 579, 329-367 (2016).

5. Castaño-Díez, D. \& Zanetti, G. In situ structure determination by subtomogram averaging. Curr. Opin. Struct. Biol. 58, 68-75 (2019).

6. Castaño-Díez, D., Kudryashev, M., Arheit, M. \& Stahlberg, H. Dynamo: A flexible, user-friendly development tool for subtomogram averaging of cryo-EM data in high-performance computing environments. J. Struct. Biol. 178, 139-151 (2012). 
7. Castaño-Díez, D., Kudryashev, M. \& Stahlberg, H. Dynamo Catalogue: Geometrical tools and data management for particle picking in subtomogram averaging of cryo-electron tomograms. J. Struct. Biol. 197, 135-144 (2017).

8. Castaño-Díez, D. The Dynamo package for tomography and subtomogram averaging: components for MATLAB, GPU computing and EC2 Amazon Web Services. Acta Crystallogr. Sect. Struct. Biol. 73, (2017).

9. Navarro, P. P., Stahlberg, H. \& Castaño-Díez, D. Protocols for Subtomogram Averaging of Membrane Proteins in the Dynamo Software Package. Front. Mol. Biosci. 5, (2018).

10. Schur, F. K. M. et al. An atomic model of HIV-1 capsid-SP1 reveals structures regulating assembly and maturation. Science aaf9620 (2016)

11. Kovtun, O. et al. Structure of the membrane-assembled retromer coat determined by cryo-electron tomography. Nature 561, 561-564 (2018).

12. Hutchings, J., et al. Subtomogram averaging of COPII assemblies reveals how coat organization dictates membrane shape. Nat. Commun. 9, 4154 (2018).

13. Watanabe, R. et al. The in situ structure of Parkinson's disease-linked LRRK2. bioRxiv 837203 (2019) 